\title{
Electron flux and pressure dynamic in the LHC vacuum pilot sector as a function of beam parameters and beam pipe properties
}

\author{
Elena Buratin $\odot,{ }^{1,2, *}$ Vincent Baglin $\odot,{ }^{1}$ Bernard Henrist, ${ }^{1}$ \\ Paolo Chiggiato, ${ }^{1}$ and Ambrogio Fasoli ${ }^{2}$ \\ ${ }^{1}$ CERN, Technology Department, Vacuum Surfaces and Coatings Group, \\ 1211-CH Geneva 23, Switzerland \\ ${ }^{2}$ Ecole Polytechnique Fédérale de Lausanne (EPFL), Swiss Plasma Center, \\ 1015 Ecublens, Lausanne, Switzerland
}

(Received 20 July 2020; accepted 8 October 2020; published 19 November 2020)

\begin{abstract}
The Large Hadron Collider (LHC) is affected by the electron cloud (EC) phenomenon that can provoke beam instabilities, detrimental heat loads and pressure increases in the vacuum system. An innovative dedicated system called vacuum pilot sector (VPS) provides a continuous monitoring of the electron flux and of the pressure signals thanks to electron pickup and vacuum gauges. The VPS system is installed in a room temperature, field-free part of the LHC storage ring. Several technical surfaces, such as ex situ nonevaporable getter (NEG), amorphous carbon coating and copper, are simultaneously tested. The main outcomes of this study show that the EC signals have: (1) a linear dependence upon the number of bunches and upon the bunch population in the multipacting regime, (2) a multipacting threshold at a given bunch population, (3) a reduction under beam conditioning, (4) a strong dependence on the filling pattern and beam energy. The comparison between different surfaces shows that amorphous carbon coating reduces drastically the EC buildup, thanks to its low secondary electron yield (SEY) and photoelectron yield (PY), while copper and ex situ NEG coated surfaces suffer of EC multipacting, even after several months of operation. The multipacting rate coefficients are higher for copper than for ex situ NEG, as predicted from the SEY estimation. Other detailed experimental observations are discussed in this paper.
\end{abstract}

DOI: 10.1103/PhysRevAccelBeams.23.114802

\section{INTRODUCTION}

The Large Hadron Collider (LHC) is a $27-\mathrm{km}$ circumference accelerator where two proton beams circulate in opposite directions [1,2]. The beam structure can host a maximum of 2820 proton bunches, spaced in time by $25 \mathrm{~ns}$, with about $10^{11}$ protons/bunch. Batches of a maximum of 72 bunches are injected in the LHC from the Super Proton Synchrotron (SPS) at an energy of $450 \mathrm{GeV}$. Once the LHC is filled, the beam energy is ramped up to $6.5 \mathrm{TeV}$ for proton collisions in the so-called stable beam phase [3,4]. A schematic of the three main phases of the LHC, i.e., the beam injection, the energy ramp-up and the stable beam, is represented in Fig. 1.

LHC beams generate electron clouds (EC) as predicted by simulations [5-8]. This phenomenon affects beam

\footnotetext{
*Corresponding author. elena.buratin@epfl.ch

Published by the American Physical Society under the terms of the Creative Commons Attribution 4.0 International license. Further distribution of this work must maintain attribution to the author(s) and the published article's title, journal citation, and DOI.
}

performance, gas density, and heat load in the cryogenic systems.

At injection energy, free electrons are generated by beam gas ionization and by beam losses along the ring.

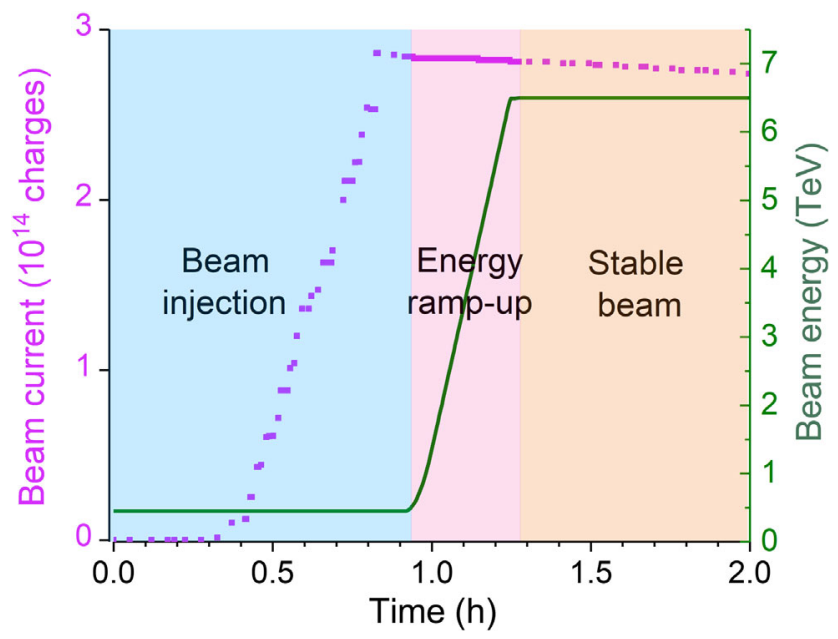

FIG. 1. A standard LHC fill during injection, energy ramp-up and stable beam phase. The beam current (circulating charges) and the beam energy $(\mathrm{GeV})$ are displayed in purple and green, respectively. 


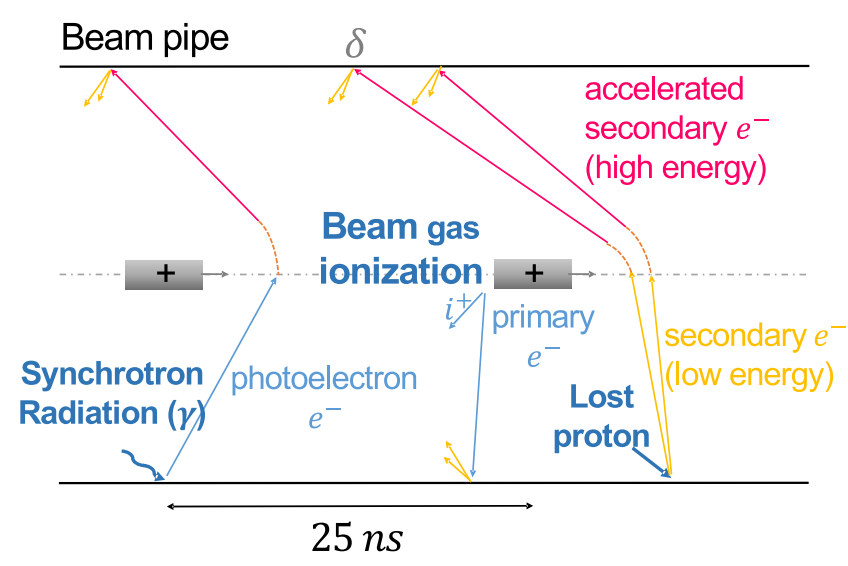

FIG. 2. Schematic of the EC buildup and multipacting process. Synchrotron radiation, beam gas ionization and lost protons generate free electrons. These electrons are accelerated by the beam electromagnetic field and impinge on the opposite beam pipe wall. They can generate secondary electrons depending on the surface SEY.

Additionally, at higher beam energy synchrotron radiation (SR) extracts photoelectrons from the inner walls of the beam pipe. The SR of LHC is typically described by a nonmonochromatic spectrum that changes with proton beam energy [9-11]. To characterize the wide energy distribution, a parameter called critical energy, $\varepsilon_{c}$, is defined. It corresponds to the photon energy that divides the SR power spectrum into two halves and it is proportional to the beam current and to the third power of the beam energy. The SR critical energy at $450 \mathrm{GeV}$ and $7 \mathrm{TeV}$ is 0.01 and $44.15 \mathrm{eV}$, respectively. To extract photoelectrons, the SR needs to have an energy above the material work function, therefore at $450 \mathrm{GeV}$ the photoelectric production does not take place. Above $2.8 \mathrm{TeV}$, the critical energy is equivalent to $3 \mathrm{eV}$ and the photoelectron production starts. The SR is also described by the photon flux, proportional to the beam current and the beam energy, and by the power, linked to the beam current and the fourth power of the beam energy.

During each bunch passage, such electrons are accelerated by the electromagnetic field associated with the beam and they hit the vacuum pipe producing secondary electrons, as depicted in Fig. 2. Bunch after bunch, a multipacting regime can be achieved. This happens when there are more than 6 to 10 bunches per batch $[12,13]$, the bunch population is above a certain threshold, and the socalled surface secondary electron yield ${ }^{1}$ (SEY, indicated as $\delta$ in the following formulas), i.e., the number of electrons extracted from the surface per impinging electron, is higher than a certain threshold, which depends on the beam structure and the geometry of the beam pipe $[6,7]$.

\footnotetext{
${ }^{1}$ In the surface science community such surface parameter is called total electron yield (TEY) if it includes the elastically reflected and backscattered electrons.
}

The SEY depends on the chemical composition of the surface and on the energy of the primary electrons. A maximum SEY $\left(\delta_{\max }\right)$ is obtained for a typical electron energy of the order of few hundreds eV $[14,15]$. The value of $\delta$ strongly decreases with the accumulated electron dose. Two mechanisms are reported to explain the influence of electron dose: the surface cleaning by electron stimulated desorption (ESD) and the graphitization of the residual carbon adsorbed onto the surface [15]. The dose at which the stable value is reached depends on the energy of the primary electrons. For an electron energy around $250 \mathrm{eV}$, an asymptotic value of SEY is obtained for an accumulated dose higher than about $10^{-2} \mathrm{C} / \mathrm{mm}^{2}$. Among the three materials, copper has the highest SEY $[14,16]$ and carbon the lowest one $[17,18]$.

The effect of the accumulated dose is used to mitigate the EC by beam scrubbing, i.e., running the accelerator so that an intense electron bombardment of the beam exposed surfaces is achieved in a safe operational mode [19].

The effects of EC were detected in the LHC by measuring the cryogenic heat loads and beam behaviors $[6,7]$. Direct measurements of the EC electron density were reported in the SPS in dedicated experiments with LHC beams [13].

In this work we describe the first direct measurements of EC in the LHC. EC was obtained by means of a dedicated setup, referred to as vacuum pilot sector (VPS) [20]. installed in a room-temperature, field-free, long straight section of the LHC at point 8 .

The VPS allows the measurements of electron flux and gas density in the beam pipe as a function of beam parameters, nature of the surface material, and accumulated electron dose. The contribution to EC of a selected number of beam parameters was measured, such as the number of bunches, the bunch population, the filling scheme and the beam energy.

\section{EXPERIMENTAL SETUP}

The VPS system extends over 18 meters of the LHC long straight section in point 8 . It consists of two parallel $80-\mathrm{mm}$ diameter beam pipes and contains four measurement stations, each 1.3 meter long, separated by in situ activated nonevaporable getter (NEG) thin-film coated chambers [21], equipped with ion pumps and NEG cartridges (see Fig. 3) [20].

Each station is composed of a 1-mm thick Oxigen-Free Electronic (OFE) $\mathrm{Cu}$ liner inserted into a dedicated vacuum chamber. Once installed, the liners underwent a mild bake out at $80^{\circ} \mathrm{C}$ for 24 hours to release part of the adsorbed water from walls and cables, and achieve a pressure lower than $10^{-9}$ mbar.

This work investigates three relevant materials for the LHC: detergent cleaned OFE copper (Cu) [14], ex situ NEG coating [21-23] and amorphous carbon coating (a-C) $[17,18]$. The former is the material exposed to the beam 


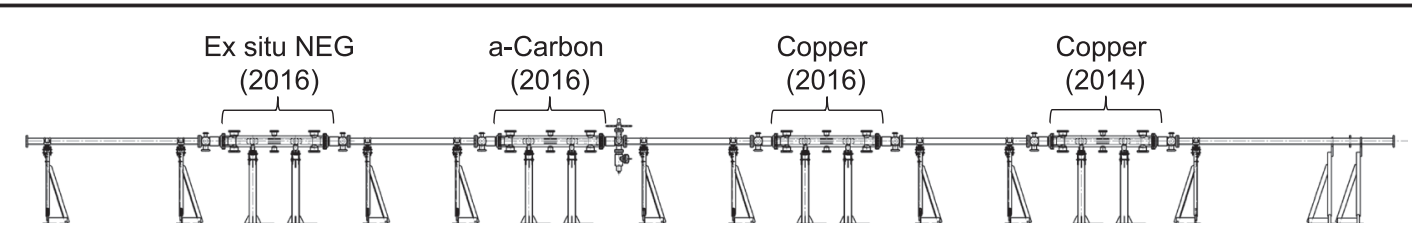

FIG. 3. Layout of the field free and room temperature VPS system, installed in the LHC ring. It is composed of four stations for the measurement of EC of different surfaces and coatings. Since 2016, ex situ NEG, a-C and copper have been installed and studied.

in the LHC's arcs and it is expected to have the highest $\delta$ among the three materials. NEG coating (1.5-2 $\mu \mathrm{m}$ thick Ti-Zr-V film) is the standard surface for the room-temperature long straight sections of the LHC [22]. The NEG coated chambers were activated in the laboratory at $230^{\circ} \mathrm{C}$ for 24 hours, vented with nitrogen and installed in the VPS without further activation. This unusual activation procedure was adopted to study the performance of this material without in situ activation, i.e., a typical situation in case of quick intervention in the beam line during the operation period. In this work, these liners are referred to as ex situ NEG [12]. The 400-600 nm thick amorphous carbon coating is a proposed EC mitigation technique for nonbakable sections of the LHC. The $\delta$ of such a coating is reported to be about $1[17,18]$.

Figure 3 shows the layout of the VPS. The first three stations were installed in 2016; the materials of the liners were ex situ NEG, a-C coating, and unbaked OFE copper. The 4th station from left to right was an additional unbaked OFE copper that was installed in 2014 and housed complementary diagnostics.

Dedicated shielded electron pickups were designed [20], manufactured and installed on the liners. These detectors are composed of a grounded grid and a collector. The grid has a transparency of 7\%; it was used to limit the effect of the measurement on the EC density (see Fig. 4) while shielding the collector from the electromagnetic field of the beam. The collector is polarized at $9 \mathrm{~V}$; this bias was applied to collect all secondary electrons extracted from the collector. Standard 50- $\Omega$ coaxial Kapton cables were installed to carry the collector signals. The vacuum flange was grounded. The signals were carried from vacuum to air

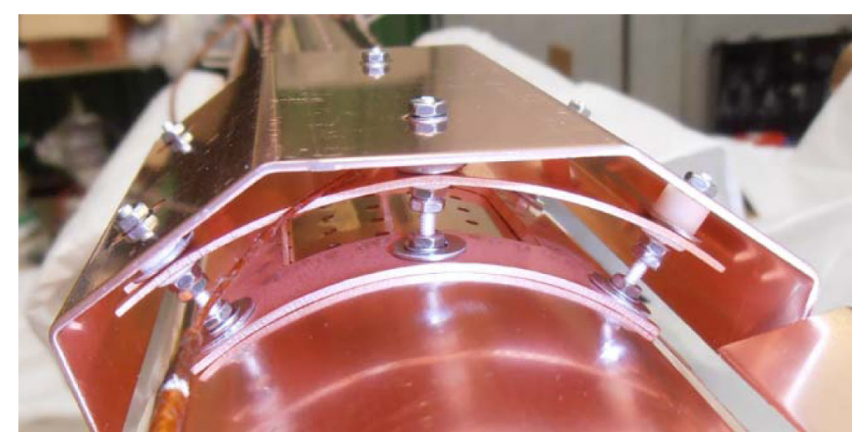

FIG. 4. Picture of a shielded pickup installed in the VPS system. Each pickup is composed of a grounded grid $(7 \%$ transparency) and a collector polarized at $9 \mathrm{~V}$. by SubMiniature version A connector (SMA) and Bayonet Neil-Concelman connector (BNC) connectors through $50-\Omega$ standard cables towards a Keithley picoammeter.

Bayard-Alpert and Penning gauges were used to measure the nitrogen equivalent total pressure along the VPS.

\section{MEASUREMENTS}

During the injection phase of a standard 25-ns beam, the influence of the number of bunches was studied, up to

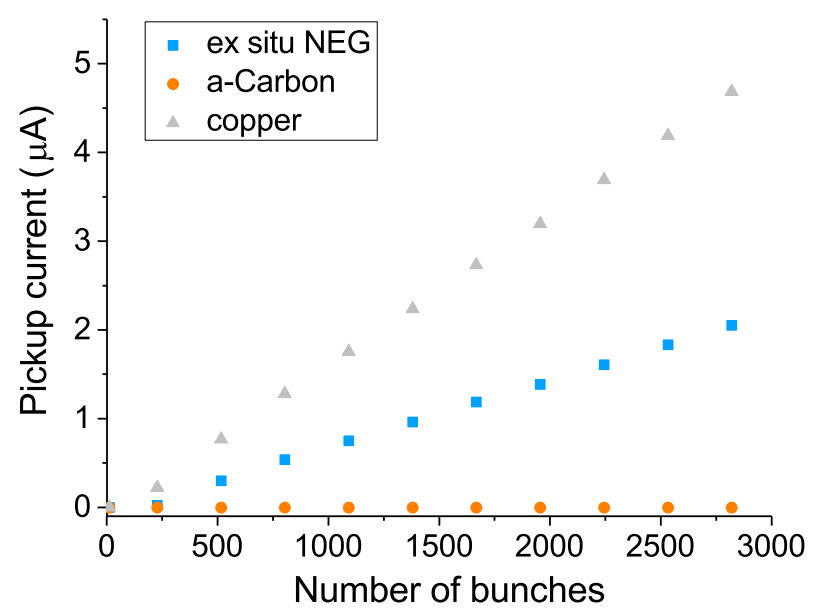

(a)

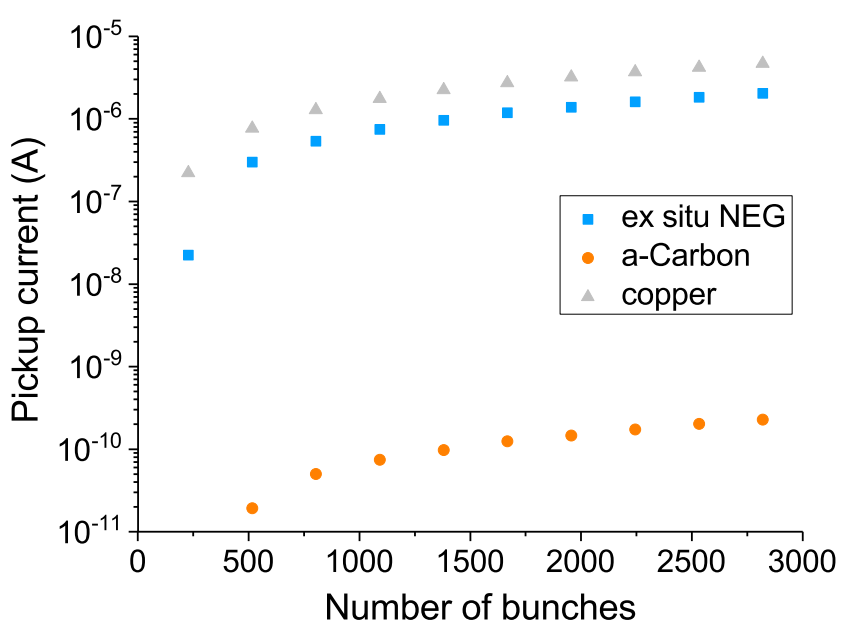

(b)

FIG. 5. Electrical signals (A) as a function of the number of bunches during the injection phase at $450 \mathrm{GeV}$ of a 25 -ns standard LHC beam in linear (a) and logarithmic (b) scales. 
TABLE I. Slopes of the EC increase as a function of the number of bunches, for the three surfaces.

\begin{tabular}{lc}
\hline \hline Surface & Slope $\left[A_{\mathrm{EC}} /\right.$ bunch $]$ \\
\hline Copper & $1.7 \times 10^{-9}$ \\
Ex situ NEG & $7.5 \times 10^{-10}$ \\
a-carbon & $8.5 \times 10^{-14}$ \\
\hline \hline
\end{tabular}

2820 bunches (Sec. III A). At the end of the injection phase, the influence of the bunch population was measured keeping the energy at $450 \mathrm{GeV}$ (Sec. III B). During the scrubbing phase, the mitigation of the EC was monitored (Sec. III C). Finally the contribution of the photoelectrons was identified for two filling schemes (Sec. III D) comparing EC measurements at injection and top energy.

\section{A. Influence of the number of bunches on EC}

A typical LHC beam (fill number 5800) is chosen to study the relationship between the EC signal and the number of bunches. This fill took place at the beginning of the 2017's LHC run, during the scrubbing period operated at $450 \mathrm{GeV}$. This beam contained 2820 bunches, with an average bunch population of $1.2 \times 10^{11}$ protons at injection. The accumulated electron doses before this fill were significantly large, around $1.5 \times 10^{-3} \mathrm{C} / \mathrm{mm}^{2}$ for the $\mathrm{Cu}$ surface, $3 \times 10^{-4} \mathrm{C} / \mathrm{mm}^{2}$ for the ex situ NEG and $1.2 \times$ $10^{-7} \mathrm{C} / \mathrm{mm}^{2}$ for the a-C surface. Each dose increase during the studied fill is much smaller than the accumulated one.

The EC signals for the three surfaces are displayed in Fig. 5 as a function of the number of bunches, $N_{b}$. Both linear and logarithmic scales are shown. The collected signals increase linearly with the number of bunches. The

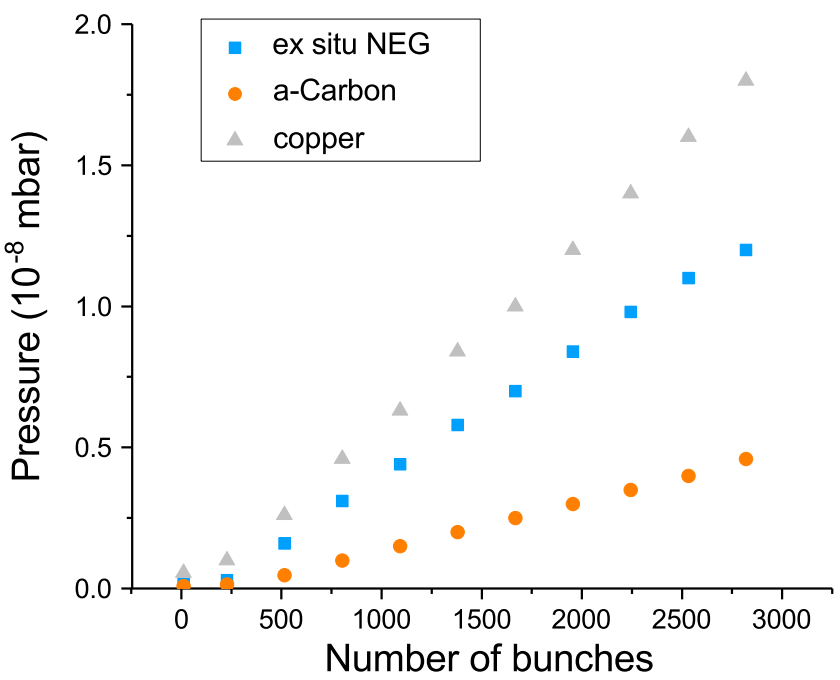

FIG. 6. Pressure trends as a function of the number of bunches during the injection phase at $450 \mathrm{GeV}$ of a 25 -ns standard LHC beam in linear scale.
TABLE II. Slopes of the pressure increase as a function of the number of bunches.

\begin{tabular}{lc}
\hline \hline Surface & Slope $[\mathrm{mbar} / \mathrm{bunch}]$ \\
\hline Copper & $6.4 \times 10^{-12}$ \\
Ex situ NEG & $4.5 \times 10^{-12}$ \\
a-carbon & $1.7 \times 10^{-12}$ \\
\hline \hline
\end{tabular}

copper station exhibits the highest EC signal, followed by a smaller signal for the ex situ NEG, and a much lower one for the a-C coated beam pipe. The linear behavior is visible in the a-C station only at the beginning of the run in Fig. 5 . As expected, the higher the SEY, the higher the EC signal.

The numerical values can be fitted by a line whose slopes are reported in Table I.

In Fig. 6, the pressure increases in the VPS stations are plotted as a function of the number of bunches. The pressures rise linearly with the number of bunches, as measured for the electron current signal.

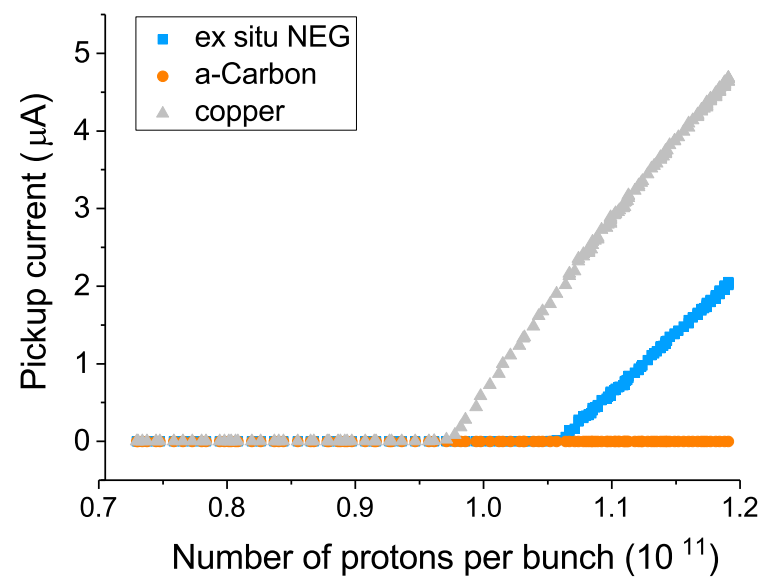

(a)

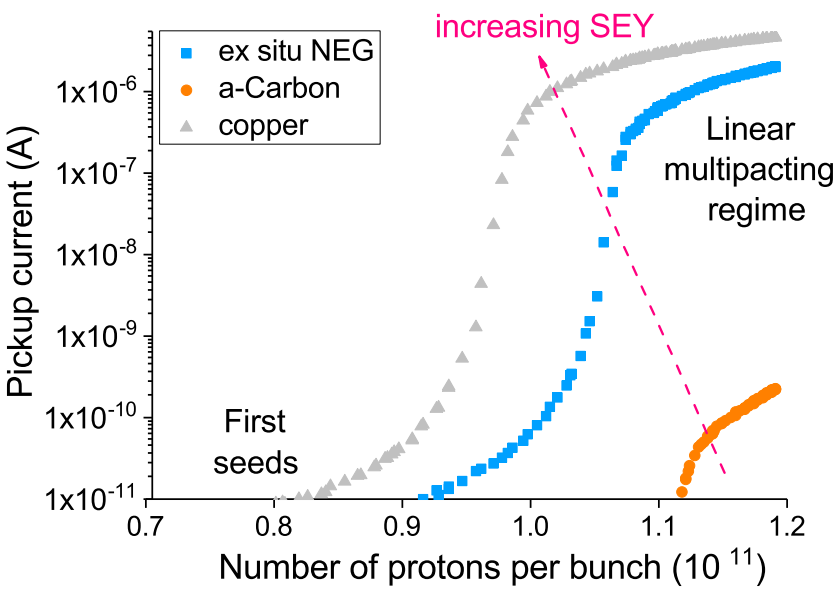

(b)

FIG. 7. Electrical signals as a function of the number of protons per bunch after the injection phase of a 25-ns standard LHC beam in linear (a) and logarithmic (b) scales at $450 \mathrm{GeV}$. 
TABLE III. Slopes of the linear EC increase as a function of the bunch population and multipacting threshold values for the three surfaces.

\begin{tabular}{lcc}
\hline \hline Surface & Slope $\left[A_{\mathrm{EC}} /\right.$ proton] & Threshold [protons/bunch] \\
\hline Copper & $2.0 \times 10^{-16}$ & $9.6 \times 10^{10}$ \\
Ex situ NEG & $1.5 \times 10^{-16}$ & $1.06 \times 10^{11}$ \\
a-carbon & $2.9 \times 10^{-20}$ & $1.12 \times 10^{11}$ \\
\hline \hline
\end{tabular}

The pressure values are fitted by simple linear equations, whose slopes are presented in Table II.

The order of the slope follows the one of the expected SEY.

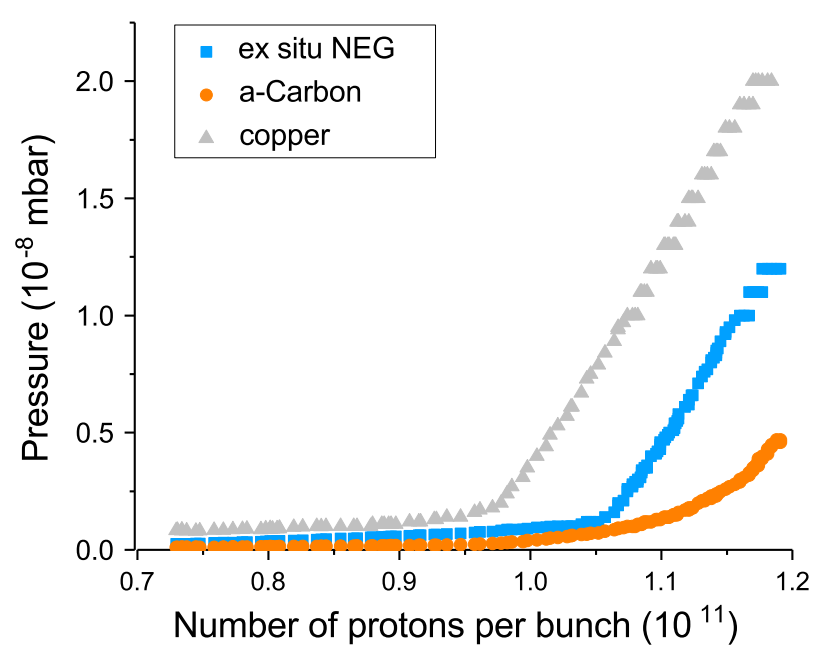

FIG. 8. Pressure trends as a function of the number of protons per bunch after the injection phase of a $25 \mathrm{~ns}$ standard LHC beam in linear at $450 \mathrm{GeV}$.

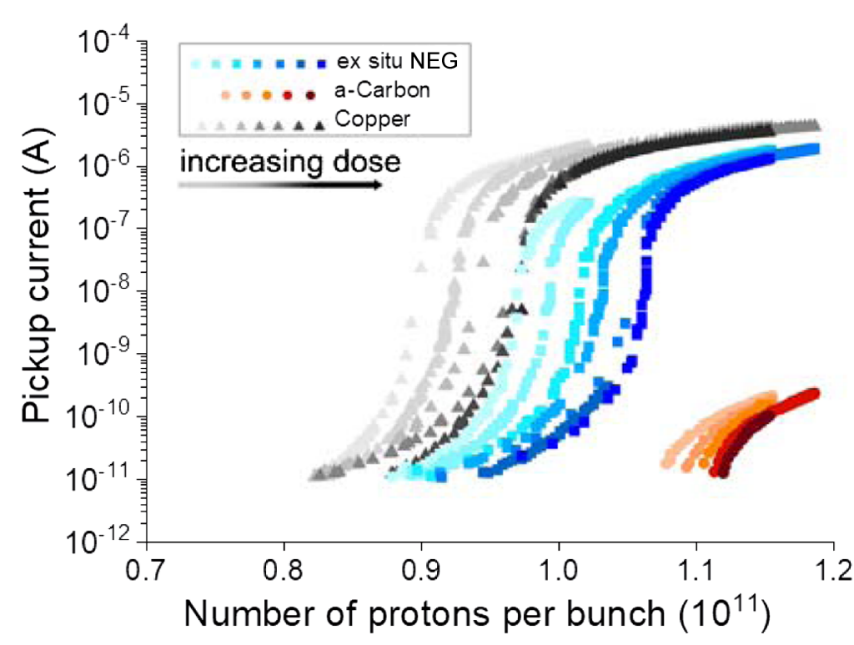

FIG. 9. Accumulated electron dose effect on the electrical signals at $450 \mathrm{GeV}$ as a function of the number of protons per bunch, in logarithmic scale.
TABLE IV. Slopes of the pressure increase as a function of the bunch population. The value for a-C coating will be discuss in the results.

\begin{tabular}{lc}
\hline \hline Surface & Slope [mbar/proton] \\
\hline Copper & $9.5 \times 10^{-19}$ \\
Ex situ NEG & $8.6 \times 10^{-19}$ \\
a-carbon & $4.4 \times 10^{-19}$ \\
\hline \hline
\end{tabular}

\section{B. Influence of the bunch population}

During the running of the LHC fill mentioned above, the bunch population decreased due to proton losses. Such a

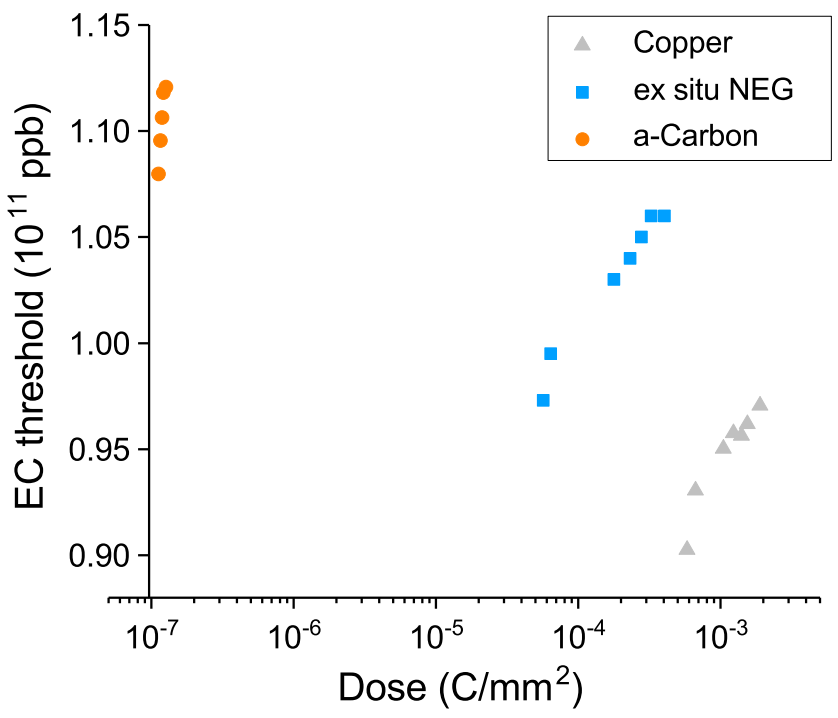

FIG. 10. Bunch population threshold to trigger electron multipacting as a function of the accumulated electron dose during the 2017's scrubbing run.

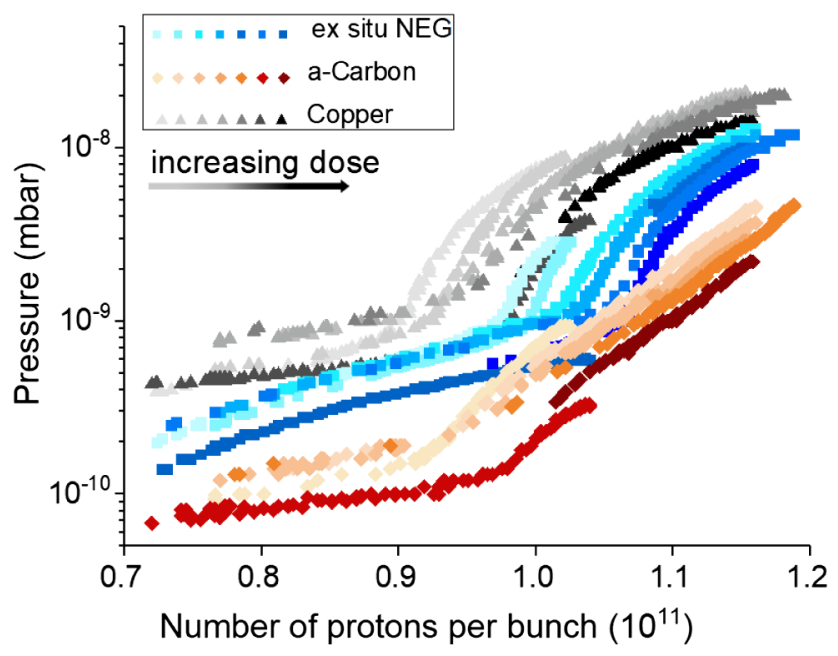

FIG. 11. Accumulated electron dose effect on the pressure trends at $450 \mathrm{GeV}$ as a function of the number of protons per bunch, in logarithmic scale. 
(a)
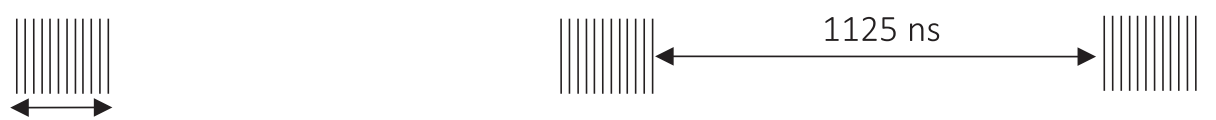

12 bunches ( 25 ns bunch spacing)

(b)
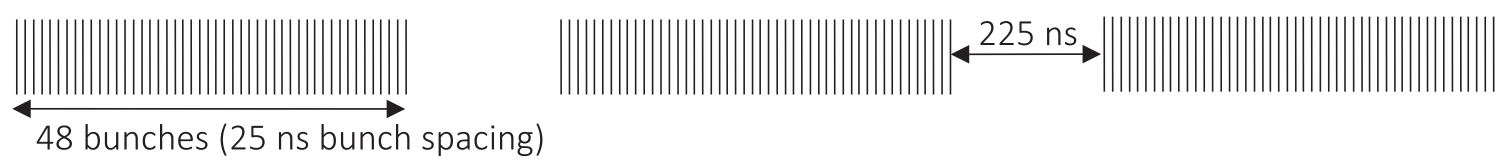

(c)
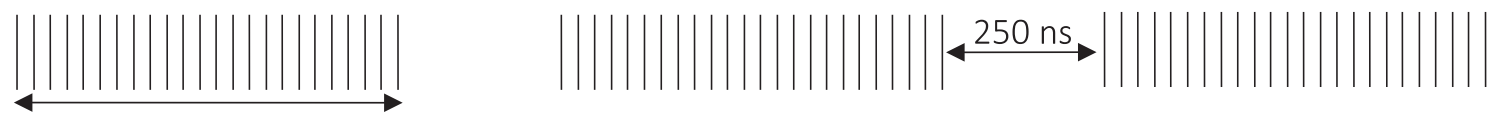

24 bunches (50 ns bunch spacing)

FIG. 12. The schemes of the first three batches are presented for the three fills: (a) fill 5717, (b) fill 5887, (c) fill 5980.

variation is exploited to correlate bunch population with the EC signals.

The initial bunch population was around $1.2 \times 10^{11}$ protons per bunch. After several hours of beam circulation, the bunch population halved. The effect of bunch population on EC is related to the beam electromagnetic field and, consequently, to the kinetic energy gained by the electrons [23].

The EC electrical currents are displayed as a function of the number of protons per bunch, $p p b$ (see Fig. 7). The three signals increase linearly, above different thresholds. Also in this case, the SEY influences significantly the magnitude of the collected current. The highest current is reached by the copper sample, followed by ex situ NEG and a-C coating. The threshold, clearly visible in linear scale, corresponds to the inflection point of the curves in the logarithmic scale. The slopes of the linear fit and the thresholds are reported in Table III.

The pressure measurements are also displayed as a function of the bunch population (see Fig. 8). The vacuum dynamics follows the same trend of the electron signal; namely, linear behavior above a certain threshold. Above the threshold, the $\mathrm{Cu}$ station has the dominant pressure signal, in the order of $10^{-8}$ mbar. The pressure thresholds (see Fig. 8) match with the electron activity thresholds (see
Fig. 7 and Table III) for $\mathrm{Cu}$ and ex situ NEG. The a-C behavior will be examined in the discussion.

The slopes of the linear fit are reported in Table IV.

\section{Influence of the accumulated electron dose}

During the 2017's scrubbing run, beams of 2820 bunches were injected in LHC (fill numbers from 5788 to 5821). The accumulated dose is calculated integrating the collected electron signal and taking into account the transparency of the grid. The scrubbing run increased the accumulated dose from $6 \times 10^{-4} \mathrm{C} / \mathrm{mm}^{2}$ to $2 \times 10^{-3} \mathrm{C} / \mathrm{mm}^{2}$ for the $\mathrm{Cu}$ surface, $6 \times 10^{-5} \mathrm{C} / \mathrm{mm}^{2}$ to $4 \times 10^{-4} \mathrm{C} / \mathrm{mm}^{2}$ for the ex situ NEG surface, and $1 \times 10^{-7} \mathrm{C} / \mathrm{mm}^{2}$ to $1.3 \times 10^{-7} \mathrm{C} / \mathrm{mm}^{2}$ for the a-C surface.

Figure 9 shows the electrical signals at different accumulated doses as a function of protons per bunch. For increasing doses, the increase of the thresholds and a slight reduction of the slopes are visible. These shifts are evident signs of the conditioning process happening at the surface.

The threshold increase can be displayed as a function of the electron dose accumulated during the scrubbing run (see Fig. 10). The three samples accumulated different electron doses, owing to the different electron activities.

TABLE V. Information related to three fills exploited in this work.

\begin{tabular}{lcccccccc}
\hline \hline & & & & & & \multicolumn{2}{c}{ Accumulated electron doses } \\
\cline { 3 - 8 } & & & & & & & \\
Funch & & Bunches & & Initial & & \\
Fill No. & spacing (ns) & Total $N_{b}$ & per batch & Total batches & ppb (protons) & Ex situ NEG & a-C \\
\hline 5717 & 25 & 75 & 12 & 12 & $1.2 \times 10^{11}$ & $1.4 \times 10^{-9}$ & $2.7 \times 10^{-11}$ & $1.9 \times 10^{-5}$ \\
5887 & 25 & 2556 & 48 & 53 & $1.16 \times 10^{11}$ & $5.8 \times 10^{-4}$ & $1.8 \times 10^{-6}$ & $3.7 \times 10^{-3}$ \\
5980 & 50 & 1284 & 24 & 53 & $1.2 \times 10^{11}$ & $6 \times 10^{-4}$ & $2 \times 10^{-6}$ & $4 \times 10^{-3}$ \\
\hline \hline
\end{tabular}


The pressure behaviors are displayed in Fig. 11 as a function of the bunch population and the accumulated electron dose. This graph shows a shift of the threshold to higher bunch populations while increasing the dose, as seen for the electrical signals (Fig. 10).

\section{Influence of the filling scheme from injection to top energy}

Along the run performed in 2017, different beams were injected in the LHC and ramped up to the top energy. Increasing the beam energy also photoelectrons contribute to the EC buildup in addition to multipacting. This additional electron emission is due to SR photons with energy higher than the typical work function of metals $(5 \mathrm{eV})$ [24].

A measurable effect of the photoelectrons was detected for proton beam energies higher than $2.8 \mathrm{TeV}$ [12] in a $50 \mathrm{~ns}$ bunch spacing beam, investigated in a previous study [12]. The effect of bunch spacing (25 and $50 \mathrm{ns)}$ was also compared. At top energy, the comparison of the collected signals obtained with the two bunch spacing allowed to disentangle the photoelectron and multipacting contribution to EC. Indeed, for a given beam current and energy, synchrotron radiation emission does not depend on the bunch spacing, while multipacting is possible only with 25 ns spacing [12].

In this study we investigate two filling schemes. Each LHC beam is composed of a pilot bunch, followed by batches of bunches. The number of bunches per batch can vary and in this work we compare fills with 12 and 48 bunches per batch. For each beam, the contribution of photoelectron is calculated normalizing to the beam current the collected signals obtained with the 50 ns beam [12]. The relevant information about the three beams is summarized in Fig. 12 and in Table V. The structure presented in Fig. 12 consists of three consecutive batches, for the three compared filling schemes: (a) the 12 bunches per batch (fill 5717), (b) the 48 bunches per batch (fill 5887), (c) the $50 \mathrm{~ns}$ bunch spacing fill with 24 bunches per batch (fill 5980). Notice that during fills 5887 and 5980 the LHC machine was full, while for the fill 5717 a total of six batches of 12 bunches were injected.

\section{Collected currents for 48 bunches per batch}

The EC and photoelectron signals are shown in Fig. 13 for the three samples. On the legends, the total electron contribution is called EC $+\mathrm{SR}$, while the photoelectron contribution is marked with SR. The beam energy and the beam current are also displayed as a function of time. Time $t=0$ is arbitrarily set at the beginning of the energy ramp-up.

During injection at $450 \mathrm{GeV}$, the EC linearly increases with the number of bunches for ex situ NEG and copper surfaces. For a-C, no EC is detected at injection energy during the fill 5887. This apparent contradiction with Fig. 5 will be clarified in the next chapter.

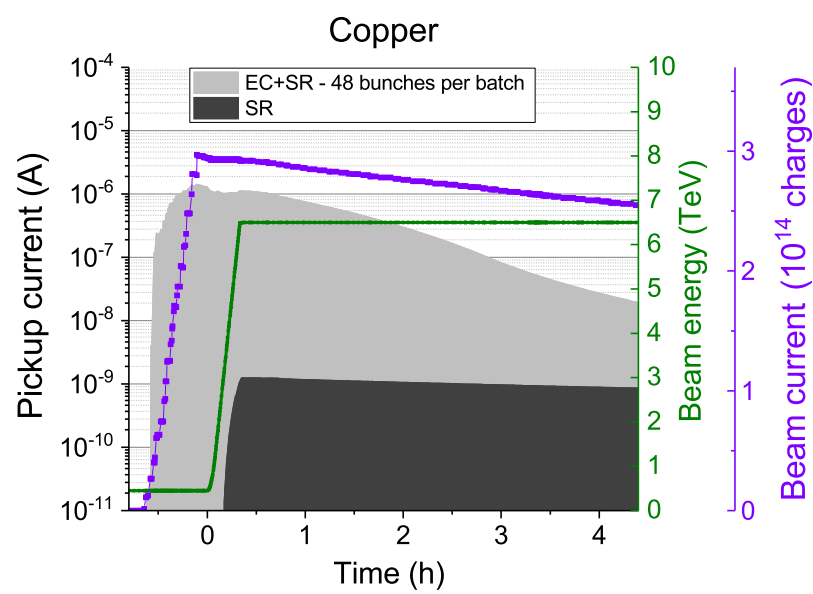

(a)

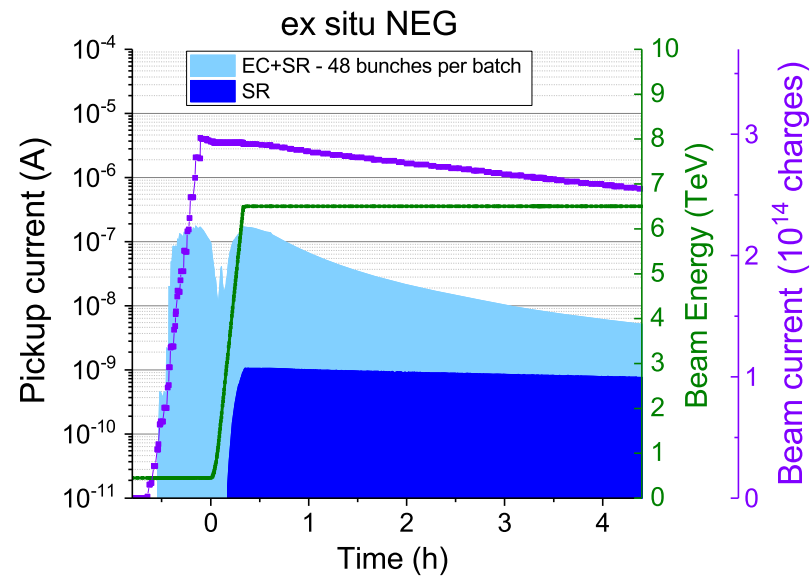

(b)

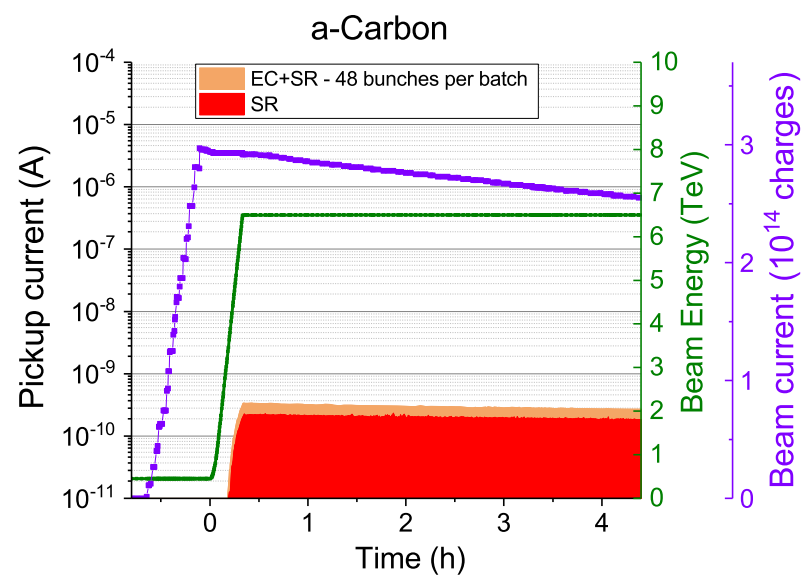

(c)

FIG. 13. EC dynamic along a standard 48 bunches per batch fill, comparing the total EC buildup with the contribution of photoelectrons for $\mathrm{Cu}$ (a), ex situ NEG (b) and a-C coating (c).

The variation of the EC signal at the beginning of the energy ramp is caused by bunch length variations due to beam setup [12]. 


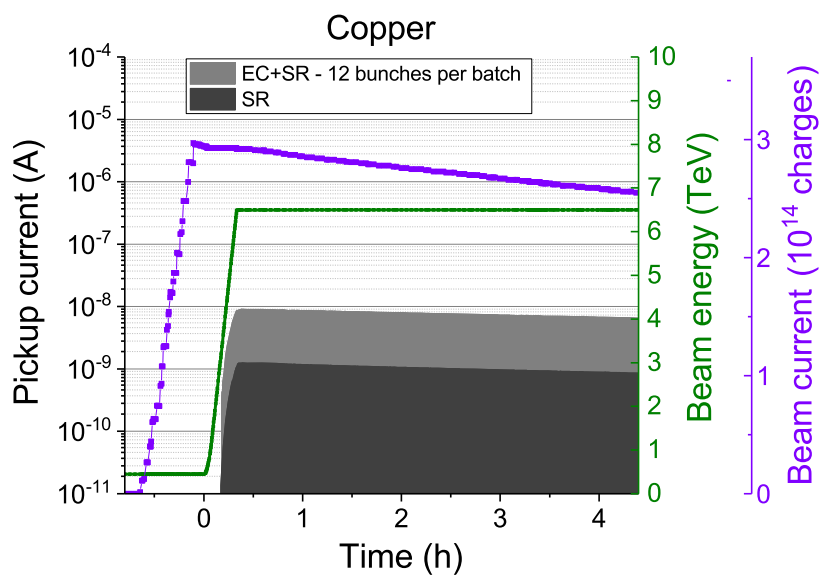

(a)

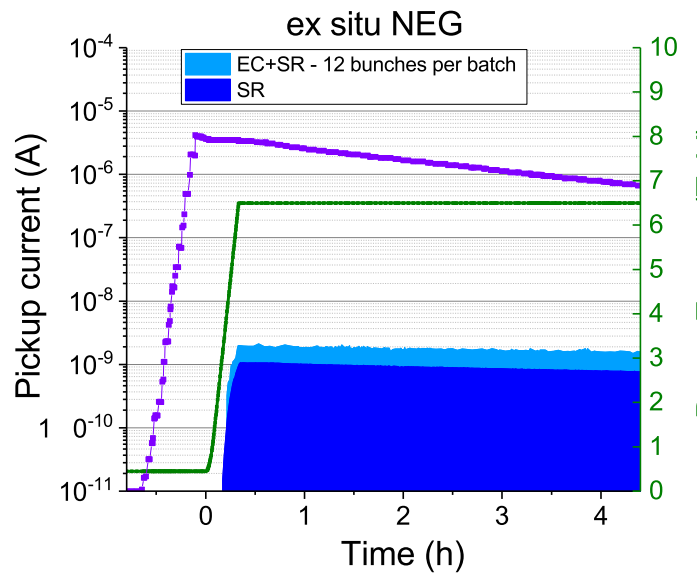

(b)

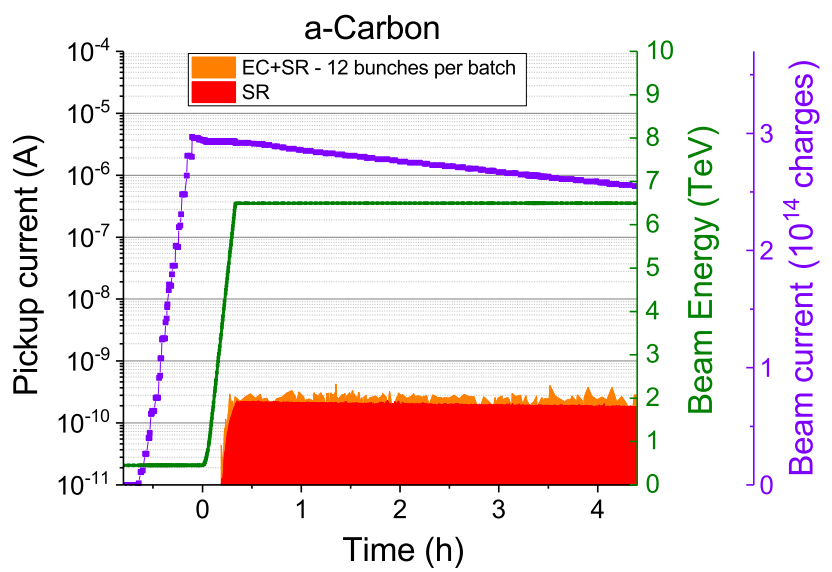

(c)

FIG. 14. EC dynamics along a 12 bunches per batch fill, comparing the total EC buildup with the effect of photoelectrons for $\mathrm{Cu}$ (a), ex situ NEG (b) and a-C coating (c).

Above $2.8 \mathrm{TeV}$, the photoelectron production becomes visible, adding a contribution to the multipacting regime in the cases of ex situ NEG and $\mathrm{Cu}$. The a-C coating shows an electrical signal for proton energies slightly higher than the threshold of photoelectron emission. This small mismatch could be attributed to a propagation of measurement errors following the normalization process or to a tiny photoelectron multipacting in a-C coated sample.

At collision energy, the collected currents from ex situ NEG and $\mathrm{Cu}$ samples decay following the bunch population evolution as explained in Sec. III B. The photoelectron current decays as the beam current.

\section{Collected currents for 12 bunches per batch}

The EC and photoelectron signals are shown in Fig. 14 for the beams made of 12 bunches per batch. At injection energy, such beam does not generate EC signals in any of the samples. This might be explained by the need of six to ten bunches for the EC buildup process to start and stabilize [6-8]. With batches of 12 bunches, the EC multipacting mechanism is interrupted as soon as generated at the end of each batch, so the cloud is not sustained and not measurable by the VPS pickups.

Above $2.8 \mathrm{TeV}$, an electrical signal starts to be detected. It is higher than the photoelectron current but follows its trend for $\mathrm{Cu}$ and ex situ NEG. For a-C, no significant differences can be reported between the photoelectron and the EC collected currents.

The equivalent analysis for the pressure variations is more complex because there is a cross talk among the pressure rises of the measurement stations. For this reason, it is not presented here. It was investigated in a previous study.

\section{DISCUSSION}

With the reported measurements, we have shown that the VPS is able to detect signals directly correlated with the electron cloud density. However, such an experimental setup has an intrinsic limitation because the characteristics of the samples change as the accumulated electron dose increases. In addition, pressure measurements suffer from gas transmission between the different stations, and gas adsorption when the operation is stopped. The latter affects mostly the results of the first fills. This is particularly relevant for the a-C sample, due to the very low electron cloud activity.

As expected from the reported SEY measurements, the copper sample is the one with the highest electron currents and pressure signals in all conditions reported in this work. The collected electron current changes linearly with the number of bunches and the bunch population above a given threshold. With the accumulated dose, the threshold increases due to SEY reduction. At injection energy, electron currents are detected with the standard filling scheme composed of 48 bunches per batch. With 12 bunches per batch, the EC signals are correlated only with photoelectron multipacting; no signal is detected for beam energy lower than $2.8 \mathrm{TeV}$.

The comparison between different surfaces shows that amorphous carbon coating reduces drastically the EC 
buildup, thanks to its intrinsically low SEY that has been reported to be around $1 \pm 0.1[16,17]$. The results of Fig. 5, obtained at the beginning of the scrubbing run, show that the signal is 4 orders of magnitude lower than the one of the other samples, but still detectable. Undetectable currents are reported in Fig. 13(c) when the integrated dose in the a$\mathrm{C}$ sample are higher. This further decrease of the e-cloud signal can be ascribed to a cleaning effect due to the small electron dose collect in the meantime, i.e., a small dose of $10^{-7} \mathrm{C} / \mathrm{mm}^{2}$ is enough to erase the detected EC signal. There is a discrepancy between the electrical signal and the pressure measurements for the a-C coating. The latter increases much more than expected from the electron current detection. As reported before, this is clearly due to gas transmission from the nearby stations and can be considered as a limitation of the VPS. This has been shown in a previous study where injections were performed explicitly to calculate gas transmission between the stations. While injecting $\mathrm{H}_{2}$, we saw a pressure reduction of a factor of 10 in nearby stations due to the aspect ratio, i.e., the pipe length divided by the beam pipe radius, and the sticking probability, i.e., the probability that molecules are trapped on the NEG buffers. For a VPS aspect ratio of 42.5 and a typical hydrogen sticking factor of $0.7 \%$, a Monte Carlo simulation gives a gas transmission ratio of 13. Therefore, our $\mathrm{H}_{2}$ transmission data are in full agreement with the simulation. For other getterable gases, with a much lower sticking factor, the expected gas transmission ratio is obviously much larger, around 1000 . However, for $\mathrm{CH}_{4}$, not pumped by NEG, the transmission is only defined by the relative pumping speed and the pressure influence is approximately a factor 3 .

Therefore, the whole pressure signal in the a-C coated sample, that is a factor of 10 lower than the $\mathrm{Cu}$ pressure, is dominated by the pressure increase in the copper samples; no pressure contribution due to EC can be ascribed to the a$\mathrm{C}$ coated sample. It is interesting to notice that the curves of $\mathrm{Cu}$ and ex situ NEG have similar slopes (Fig. 11) even if the accumulated dose and the SEY parameter are different.

The filling scheme does not affect the signals collected in the a-C samples, because they depend only on the SR emitted by the beam. It is important to note that the photoelectron current in the a-C coated sample is about 5 times lower than the one measured in the copper one. This is in agreement with the lower photon electron yield (PY) of the carbon material. In particular, the PY can be estimated by the measurements performed with 50 ns (fill number 5980). For this purpose, we suppose that the PY conditioning is negligible. From the photoelectron currents, it is possible to calculate the electron flux and, consequently, estimate the PY. The values are presented in Table VI. The calculated PY of the a-C coated sample is 5 times lower than the one of conditioned copper. These values are compatible with experimental results. The accumulated doses for the three surfaces are reported in Table V (fill 5980).
TABLE VI. PY estimation for the three surfaces for $25 \mathrm{~ns}$ fill.

\begin{tabular}{lcc}
\hline \hline Surface & Photoelectron currents (A) & PY \\
\hline Ex situ NEG & $1.06 \times 10^{-9}$ & 0.04 \\
a-C & $2.16 \times 10^{-10}$ & 0.01 \\
$\mathrm{Cu}$ & $1.25 \times 10^{-9}$ & 0.05 \\
\hline \hline
\end{tabular}

The ex situ NEG sample shows EC signals at least a factor of 2 lower than copper and higher threshold for EC buildup. This benefit can be related to a lower SEY than the one of copper.

The contribution of photoelectrons at high beam energy influences the EC multipacting. Even if the SEY is around 1 , the photoelectrons trigger the electron cloud signal. Instead, if the SEY is very high, as for $\mathrm{Cu}$, the $\mathrm{EC}$ multipacting is already so large at $450 \mathrm{GeV}$ that a few more electron seeds due to SR do not influence the collected electron current (see Fig. 13).

\section{CONCLUSIONS}

The vacuum pilot sector (VPS) was designed to study electron multipacting and synchrotron radiation effects on EC activity in a room temperature field-free section of the LHC. Since the installation in 2016, the VPS performed reliable measurements during the last part of LHC run 2. The goal was to compare the behavior of ex situ NEG and amorphous-carbon coating with well-known unbaked copper samples.

For the NEG coated samples, it has been shown that the ex situ activation has a beneficial effect on the EC even if the pumping properties of the getter layers are lost due to storage in nitrogen and short venting in air before installation. Such a nonstandard NEG activation procedure could be used in case of urgent interventions that does not allow in situ bakeout cycles during operational phases.

This study confirms that the EC is drastically reduced by a-C coating, thanks to the low SEY of its surface. This result contributed to the decision to coat with a-C the beam screen of the inner triplet magnets of the LHC in the frame of the high luminosity HLC (HL-LHC) project. In addition, the very low $\mathrm{PY}$ of a-C indicated that this material can be a good candidate for coating the vacuum chamber of future positron rings, in particular the one studied in the frame of the Future Circular Collider (FCC-ee).

The VPS has been recently equipped with calorimetric detectors and analyzers for the measurement of electron energy spectra. The results of these additional measurement techniques will be reported in a future contribution.

\section{ACKNOWLEDGMENTS}

The authors acknowledge the help of M. Gasior and M. Wendt for the upgrade of the EC pickups, and J. Wenninger for the understanding of the bunch length dynamic. This 
work would not have been possible without the cooperation of many of CERN's colleagues involved in the design, the installation and the maintenance of the VPS.

[1] O. Brüning, P Collier, P. Lebrun, S. Myers, R. Ostojic, J. Poole, and P. Proudlock, Large Hadron Collider (LHC) design report, CERN Yellow Report No. 1, Geneva, 2004.

[2] L. Evans and P. Bryant, LHC machine, J. Instrum. 3, S08001 (2008).

[3] T.S. Virdee, The LHC project: The accelerator and the experiments, Nucl. Instrum. Methods Phys. Res., Sect. A 623, 1 (2010).

[4] J. Wenninger, Operation and configuration of the LHC in run 2, Report No. CERN-ACC-NOTE 0007, 2019.

[5] F. Zimmermann, Electron cloud effects in the LHC, Mini Workshop on Electron Cloud Simulations for Proton and Positron Beams (CERN, Geneva, Switzerland, 2002), pp. 47-55.

[6] G. Iadarola, Electron cloud studies for CERN particle accelerators and simulation code development, Report No. CERN-THESIS 047, 2014.

[7] G. Rumolo and F. Zimmermann, Electron cloud simulations: Beam instabilities and wakefields, Phys. Rev. ST Accel. Beams 5, 121002 (2002).

[8] E. Buratin, V. Baglin, and B. Henrist, Preliminary results obtained with the LHC vacuum pilot sector, in Proceedings of the Joint INFN-CERN-ARIES Workshop on ElectronCloud Effects (ECLOUD'18), 3-7 June 2018, La Biodola, Isola d'Elba, Italy, Vol. 7 (2020), https://doi.org/10.23732/ CYRCP-2020-007.

[9] J. Jackson, Classical Electrodynamics, 3rd d. (Wiley, New York, 1999).

[10] V. Baglin, G. Bregliozzi, J. M. Jimenez, and G. Lanza, Synchrotron radiation in the LHC vacuum system, in Proceedings of the 2nd International Particle Accelerator Conference, San Sebastián, Spain (EPS-AG, Spain, 2011).

[11] G. Arduini, K. Cornelis, O. Gröbner, N. Hilleret, W. Hofle, J. M. Jimenez, J. M. Laurent, G. Moulard, M. Pivi, and K. Weiss, Electron-cloud: Observations with LHC-type beams in the SPS, in Proceedings of the European Particle Accelerator Conference, Vienna, 2000 (EPS, Geneva, 2000).

[12] A. Hofmann, The physics of synchrotron radiation, Cambridge Monographs on Particle Physics, Nuclear
Physics and Cosmology (Cambridge University, Cambridge, England, 2004).

[13] V. Baglin, J. Bajko, O. Gröbner, B. Henrist, N. Hilleret, C. Scheuerlein, and M. Taborelli, The secondary electron yield of technical materials and its variation with surface treatments, LHC Project Report No. 443, 2000.

[14] R. Cimino, I. R. Collins, M. A. Furman, M. Pivi, F. Ruggiero, and F. Zimmermann, Can Low-Energy Electrons Affect High-Energy Physics Accelerators?, Phys. Rev. Lett. 93, 014801 (2004).

[15] R. Larciprete, D. R. Grosso, M. Commisso, R. Flammini, and R. Cimino, The chemical origin of SEY at technical surfaces, AIP Conf. Proc. C 1206051, 99 (2013).

[16] P. C. Pinto et al., Carbon coatings with low secondary electron yield, Vacuum 98, 29 (2013).

[17] C. Y. Vallgren et al., Amorphous carbon coatings for the mitigation of electron cloud in the CERN Super Proton Synchrotron, Phys. Rev. ST Accel. Beams 14, 071001 (2011).

[18] O. Brüning, F. Caspers, I. R. Collins, O. Gröbner, B. Henrist, and N. Hilleret, Electron cloud and beam scrubbing in the LHC, in Proceedings of the 18th Particle Accelerator Conference, New York, 1999 (IEEE, New York, 1999).

[19] B. Henrist, V. Baglin, G. Bregliozzi, and P. Chiggiato, The LHC vacuum pilot sector project, in Proceedings of IPAC2014, Dresden, Germany, 2014 [Report No. CERN-ACC-2014-0264, Geneva].

[20] C. Benvenuti, P. Chiggiato, F. Cicoira, and Y. L'Aminot, Nonevaporable getter films for ultrahigh vacuum applictions, J. Vac. Sci. Technol. A 16, 148 (1998).

[21] P. Chiggiato and P. Costa Pinto, Ti-Zr-V nonevaporable getter films: From development to large scale production for the Large Hadron Collider, Thin Solid Films 515, 382 (2006).

[22] B. Henrist, N. Hilleret, C. Scheuerlein, and M. Taborelli, The secondary electron yield of TiZr and TiZrV nonevaporable getter film coatings, Appl. Surf. Sci. 172, 95 (2001).

[23] J.S. Berg, Energy gain in an electron cloud during the passage of a bunch, LHC Project Note, 1997.

[24] P. O. Gartland, S. Berge, and B. J. Slagsvold, Recommended values of clean metal surface work functions, Phys. Rev. Lett. 28, 738 (1972). 